\title{
Lista actualizada de los murciélagos (Mammalia, Chiroptera) de Guatemala
}

\author{
Updated list of the bats (Mammalia, Chiroptera) of Guatemala \\ Cristian Kraker-Castañeda ${ }^{a, b, *}$, Sergio G. Pérez ${ }^{c}$, José O. Cajas-Castillo ${ }^{b}$ \\ y José L. Echeverría-Tello ${ }^{\text {b,d }}$ \\ a Departamento de Conservación de la Biodiversidad, El Colegio de la Frontera Sur, Carretera Panamericana y Periférico Sur s/n, Barrio María Auxiliadora, \\ San Cristóbal de Las Casas, 29290, Chiapas, México \\ ${ }^{\mathrm{b}}$ Escuela de Biología, Universidad de San Carlos de Guatemala, Edificio T-10, Segundo nivel, Ciudad Universitaria, Zona 12, 01010, Guatemala, Guatemala \\ ${ }^{\mathrm{c}}$ Museo de Historia Natural, Universidad de San Carlos de Guatemala, calle Mariscal Cruz 1-56, Zona 10, 01012, Guatemala, Guatemala \\ d Oficina Técnica de Biodiversidad, Consejo Nacional de Áreas Protegidas, 5ta. Avenida 6-06, zona 1, 01001, Guatemala, Guatemala
}

Recibido el 4 de julio de 2015; aceptado el 5 de octubre de 2015

Disponible en Internet el 10 de mayo de 2016

\begin{abstract}
Resumen
A partir de la revisión de bases de datos de colecciones zoológicas, literatura especializada y publicaciones científicas, presentamos una lista de murciélagos para Guatemala compuesta por 100 especies. Se incluyen 2 registros nuevos para el país (Micronycteris minuta y Carollia castanea), basados en ejemplares de colección. El incremento del trabajo de campo y estudios taxonómicos obligan a la actualización periódica de esta información para proveer conteos de especies precisos, con implicaciones en las estrategias de conservación.

Derechos Reservados @ 2016 Universidad Nacional Autónoma de México, Instituto de Biología. Este es un artículo de acceso abierto distribuido bajo los términos de la Licencia Creative Commons CC BY-NC-ND 4.0.
\end{abstract}

Palabras clave: Conservación; Guatemala; Chiroptera; Riqueza; Taxonomía

\begin{abstract}
Based on a revision of zoological collections data bases, specialized literature and scientific papers, we present a bat list for Guatemala composed by 100 species. We include 2 new records for the country (Micronycteris minuta and Carollia castanea), which are based on voucher specimens. Growing field work and taxonomic research involve periodically updating this information in order to provide precise species counts, with implications in conservation strategies.

All Rights Reserved (c) 2016 Universidad Nacional Autónoma de México, Instituto de Biología. This is an open access item distributed under the Creative Commons CC License BY-NC-ND 4.0.
\end{abstract}

Keywords: Conservation; Guatemala; Chiroptera; Richness; Taxonomy

\section{Introducción}

Publicaciones recientes se contradicen en cuanto al conteo total de especies de murciélagos para Guatemala (MacCarthy y Pérez, 2006; Méndez, 2008; Pérez, López y MacCarthy, 2012), lo que ha provocado confusión en el uso de esta información.

\footnotetext{
* Autor para correspondencia.

Correo electrónico: ckraker@ecosur.edu.mx (C. Kraker-Castañeda).

La revisión por pares es responsabilidad de la Universidad Nacional Autónoma de México.
}

MacCarthy y Pérez (2006) presentan la lista de especies de mamíferos terrestres más completa para el país basada en ejemplares de referencia de colecciones científicas y registros confiables de la literatura; en esta, se registran 95 especies de murciélagos. Estos autores sugieren la exclusión de 2 especies con registros históricos y de presencia dudosa en el país, Macrotus waterhousii Gray, 1843 y Leptonycteris nivalis (Saussure, 1860); asimismo indican especies con posible presencia basada en sus rangos de distribución y registros en países vecinos. Posteriormente, Pérez et al. (2012) confirman la presencia de 5 de las especies consideradas como posibles: Trinycteris nicefori 
(Sanborn, 1949), Thyroptera tricolor Spix, 1823, Eumops glaucinus (Wagner, 1843), Eptesicus brasiliensis (Desmarest, 1819) y Myotis thysanodes Miller, 1897; sin embargo, estos autores presentan un recuento erróneo de 104 especies. Actualmente, se cuenta con 2 registros nuevos para el país, Micronycteris minuta (Gervais, 1856) y Carollia castanea H. Allen, 1890, los cuales están basados en ejemplares depositados en la colección mastozoológica del Museo de Historia Natural, Universidad de San Carlos de Guatemala.

Recientemente, para varias de las especies de murciélagos enlistados para Guatemala se han propuesto cambios taxonómicos (e.g., Baird, Marchán-Rivadeneira, Pérez y Baker, 2012; Clare et al., 2013; Mantilla-Meluk, 2014; Velazco y Patterson, 2014; Velazco y Simmons, 2011). Las estrategias de conservación requieren conteos precisos y el incremento en el número de géneros y especies de murciélagos neotropicales (Solari y Martínez-Arias, 2014) obligan a la actualización periódica de esta información.

\section{Materiales y métodos}

Para elaborar la lista de especies se tomó como punto de partida el conteo de MacCarthy y Pérez (2006). Posteriormente, se procedió a consultar la base de datos de la colección del Museo de Historia Natural, Universidad de San Carlos de Guatemala, capítulos de libros especializados y publicaciones científicas. Para la nomenclatura y arreglo taxonómico se siguió a Simmons (2005), para lo que se consideraron los cambios taxonómicos posteriores y registros recientes. El estado de conservación de las especies corresponde a los de la Unión Internacional para la Conservación de la Naturaleza (UICN) (UICN, 2015), clasificados en las siguientes categorías, junto con sus acrónimos en inglés: no evaluado (NE), datos deficientes (DD), preocupación menor (LC), casi amenazado (NT) y vulnerable (VU). Adicionalmente, incluimos una categoría denominada no asignable (NA) para casos en los que no se tiene resuelta la situación taxonómica de la especie. La información referente a nivel de endemismo —en adelante NEn — se basó en el sistema de información geográfica de la UICN y se complementó con publicaciones científicas. Con esta información se establecieron las siguientes categorías subjetivas por su practicidad: endémica nacional $(\mathrm{EN})$, endémica regional (ER), distribución amplia (DA). Como ER, se define aquí una especie con rango de distribución cercano al límite norte del istmo de Tehuantepec y el norte de Colombia; y como DA, aquellas que sobrepasan dichos límites en una o ambas direcciones, hacia el norte y/o el sur, incluyendo el Caribe.

\section{Resultados}

A partir de las revisiones, el recuento de murciélagos para Guatemala es de 100 especies, distribuidas en 8 familias, 10 subfamilias, 6 tribus y 54 géneros (tabla 1). Adicionalmente, en la lista incluimos subespecies indicando las fuentes donde se puede encontrar información sobre sus límites de distribución geográfica y contexto taxonómico (tabla 1). Según la UICN, el número de especies por categoría de amenaza es el siguiente: NE (8), DD (1), LC (85), NT (3), VU (2); además, indicamos una especie como NA. Del total, una especie destaca como EN, Myotis cobanensis Goodwin, 1955; sin embargo, requiere una revisión profunda y esfuerzos mayores de investigación. Del resto, 18 especies se consideran ER, 80 de DA y una, Myotis auriculus Baker y Stains, 1955, NA. En la tabla 1 se presenta la lista de especies junto con información sobre su estado de conservación y nivel de endemismo.

\section{Discusión}

\section{Familia Phyllostomidae Gray, 1825}

Para esta familia, MacCarthy y Pérez (2006) incluían a Vampyressa pusilla (Wagner, 1843) (Stenodermatinae, Stenodermatini) y Sturnira ludovici Anthony, 1924 (Stenodermatinae, Sturnirini), actualmente restringidas a Sudamérica (Simmons, 2005; Velazco y Patterson, 2013). En esta lista, son tratadas como V. thyone Thomas, 1909 y S. hondurensis Goodwin, 1940, respectivamente (Ramírez-Pulido, González-Ruiz, Gardner y Arroyo-Cabrales, 2014); esta última, era considerada una subespecie de S. ludovici (Velazco y Patterson, 2013, 2014). Por otro lado Sturnira lilium (E. Geoffroy, 1810) (Stenodermatinae, Sturnirini), que anteriormente era considerada de distribución muy amplia desde México hasta Sudamérica, representa un complejo de especies (Ditchfield, 2000; Velazco y Patterson, 2013) dentro del cual la subespecie $S$. l. parvidens Goldman, 1917, cuyo rango geográfico abarca Centroamérica (Velazco y Patterson, 2014), se sugería debía ser elevada a la categoría de especie, mientras que S. lilium queda restringida a Sudamérica (Velazco y Patterson, 2013). En esta lista se incluye a $S$. parvidens Goldman, 1917.

También MacCarthy y Pérez (2006) incluyen en su lista a Artibeus intermedius J. A. Allen, 1897 (Stenodermatinae, Stenodermatini) y Vampyrodes caraccioli (Thomas, 1889) (Stenodermatinae, Stenodermatini). Actualmente, A. intermedius se considera coespecífica de A. lituratus (Olfers, 1818) basado en evidencia molecular (Hoofer, Solari, Larsen, Bradley y Baker, 2008; Redondo, Brina, Silva, Ditchfield y Santos, 2008), por lo que es excluida de esta lista. Por su parte, para $V$. caraccioli se reconocían 2 subespecies: V. caraccioli caraccioli (Thomas, 1889) y V. caraccioli major G.M. Allen, 1908 (Velazco, Aires, Carmignotto y Bezerra, 2010; Willis, Willig y Knox-Jones, 1990); la distribución de esta última abarca desde el sur de México hacia Sudamérica y actualmente es tratada como una especie aparte (Ramírez-Pulido et al., 2014; Solari y MartínezArias, 2014; Velazco y Simmons, 2011) e incluida en esta lista como V. major G.M. Allen, 1908.

Recientemente, Mantilla-Meluk (2014) publicó una revisión del género Uroderma (Stenodermatinae, Stenodermatini), en la cual propone la división de $U$. bilobatum Peters, 1866 en 2 especies que son incluidas en esta lista: Uroderma davisi Baker y McDaniel, 1972, cuyo rango de distribución se extiende a lo largo de la vertiente del Pacífico y U. convexum Lyon, 1902 en la vertiente del Atlántico. Además, MacCarthy y Pérez (2006) incluían en su lista a la subespecie Leptonycteris curasoae yerbabuenae Martínez y Villa, 1940 (Glossophaginae, Glossophagini) y en esta lista se incluye como L. yerbabuenae Martínez y Villa, 1940 siguiendo a Simmons (2005), mientras que 
Tabla 1

Lista taxonómica de las especies de murciélagos de Guatemala.

\begin{tabular}{|c|c|c|c|c|}
\hline Núm. & Categoría taxonómica & UICN & NEn & Fuente \\
\hline & Familia Emballonuridae Gervais, 1855 & & & \\
\hline & Subfamilia Emballonurinae Gervais, 1855 & & & \\
\hline 1 & Balantiopteryx io Thomas, 1904 & VU & ER & \\
\hline \multirow[t]{2}{*}{2} & Balantiopteryx plicata Peters, 1867 & $\mathrm{LC}$ & DA & \\
\hline & Balantiopteryx plicata plicata Peters, 1867 & & & Arroyo-Cabrales y Knox-Jones (1988) \\
\hline 3 & Centronycteris centralis Thomas, 1912 & $\mathrm{LC}$ & DA & \\
\hline \multirow[t]{2}{*}{4} & Diclidurus albus Wied-Neuwied, 1820 & $\mathrm{LC}$ & DA & \\
\hline & Diclidurus albus virgo Thomas, 1903 & & & Ceballos y Medellín (1988) \\
\hline \multirow[t]{2}{*}{5} & Peropteryx kappleri Peters, 1867 & $\mathrm{LC}$ & DA & \\
\hline & Peropteryx kappleri kappleri Peters, 1867 & & & Castro-Arellano y Marce (2014) \\
\hline 6 & Peropteryx macrotis (Wagner, 1843) & $\mathrm{LC}$ & DA & \\
\hline 7 & Rhynchonycteris naso (Wied-Neuwied, 1820) & $\mathrm{LC}$ & DA & \\
\hline 8 & Saccopteryx bilineata $($ Temminck, 1838) & $\mathrm{LC}$ & DA & \\
\hline \multirow[t]{3}{*}{9} & Saccopteryx leptura (Schreber, 1774) & $\mathrm{LC}$ & DA & \\
\hline & Familia Phyllostomidae Gray, 1825 & & & \\
\hline & Subfamilia Desmodontinae Bonaparte, 1845 & & & \\
\hline \multirow[t]{2}{*}{10} & Desmodus rotundus (E. Geoffroy, 1810) & $\mathrm{LC}$ & DA & \\
\hline & Desmodus rotundus murinus Wagner, 1840 & & & Greenhall, Joermann, Schmidt y Seidel (1983) \\
\hline \multirow[t]{4}{*}{11} & Diphylla ecaudata Spix, 1823 & $\mathrm{LC}$ & DA & \\
\hline & Diphylla ecaudata centralis Thomas, 1903 & & & Greenhall, Schmidt y Joermann (1984) \\
\hline & Subfamilia Glossophaginae Bonaparte, 1845 & & & \\
\hline & Tribu Glossophagini Bonaparte, 1845 & & & \\
\hline \multirow[t]{2}{*}{12} & Anoura geoffroyi Gray, 1838 & $\mathrm{LC}$ & DA & \\
\hline & Anoura geoffroyi lasiopyga (Peters, 1868) & & & Ortega y Alarcón (2008) \\
\hline 13 & Choeroniscus godmani (Thomas, 1903) & $\mathrm{LC}$ & DA & \\
\hline 14 & Choeronycteris mexicana Tschudi, 1844 & NT & DA & \\
\hline \multirow[t]{2}{*}{15} & Glossophaga commissarisi Gardner, 1962 & $\mathrm{LC}$ & DA & \\
\hline & Glossophaga commissarisi commissarisi Gardner, 1962 & & & Webster y Knox-Jones (1993) \\
\hline 16 & Glossophaga leachii Gray, 1844 & $\mathrm{LC}$ & ER & \\
\hline \multirow[t]{2}{*}{17} & Glossophaga soricina (Pallas, 1766) & $\mathrm{LC}$ & $\mathrm{DA}$ & \\
\hline & Glossophaga soricina handleyi Webster y Jones, 1980 & & & Álvarez, Willig, Knox-Jones y Webster (1991) \\
\hline \multirow[t]{2}{*}{18} & Hylonycteris underwoodi Thomas, 1903 & $\mathrm{LC}$ & ER & \\
\hline & Hylonycteris underwoodi underwoodi Thomas, 1903 & & & Knox-Jones y Homan (1974) \\
\hline 19 & Leptonycteris yerbabuenae Martínez y Villa-R., 1940 & VU & DA & \\
\hline \multirow[t]{2}{*}{20} & Lichonycteris obscura Thomas, 1895 & $\mathrm{LC}$ & DA & \\
\hline & Subfamilia Phyllostominae Gray, 1825 & & & \\
\hline \multirow[t]{2}{*}{21} & Chrotopterus auritus (Peters, 1856) & $\mathrm{LC}$ & DA & \\
\hline & Chrotopterus auritus auritus (Peters, 1856) & & & Medellín (1989) \\
\hline 22 & Lampronycteris brachyotis (Dobson, 1879) & $\mathrm{LC}$ & DA & \\
\hline \multirow[t]{2}{*}{23} & Lonchorhina aurita Tomes, 1863 & $\mathrm{LC}$ & DA & \\
\hline & Lonchorhina aurita aurita Tomes, 1863 & & & Ramírez-Pulido et al. (2014) \\
\hline 24 & Lophostoma brasiliense Peters, 1866 & $\mathrm{LC}$ & DA & \\
\hline 25 & Lophostoma evotis (Davis y Carter, 1978) & $\mathrm{LC}$ & ER & \\
\hline 26 & Macrophyllum macrophyllum (Schinz, 1821) & $\mathrm{LC}$ & DA & \\
\hline \multirow[t]{2}{*}{27} & Micronycteris microtis Miller, 1898 & $\mathrm{LC}$ & DA & \\
\hline & Micronycteris microtis mexicana Miller, 1898 & & & Alonso-Mejía y Medellín (1991) \\
\hline 28 & Micronycteris minuta (Gervais, 1856) & $\mathrm{LC}$ & DA & \\
\hline 29 & Micronycteris schmidtorum Sanborn, 1935 & $\mathrm{LC}$ & DA & \\
\hline 30 & Mimon cozumelae Goldman, 1914 & $\mathrm{LC}$ & ER & \\
\hline \multirow[t]{2}{*}{31} & Mimon crenulatum (E. Geoffroy, 1803) & $\mathrm{LC}$ & DA & \\
\hline & Mimon crenulatum keenani Handley, 1960 & & & Williams y Genoways (2007) \\
\hline 32 & Phylloderma stenops Peters, 1865 & $\mathrm{LC}$ & DA & \\
\hline & Phylloderma stenops septentrionalis Goodwin, 1940 & & & Williams y Genoways (2007) \\
\hline 33 & Phyllostomus discolor Wagner, 1843 & $\mathrm{LC}$ & DA & \\
\hline & Phyllostomus discolor verrucosus (Elliot, 1905) & & & Kwiecinski (2006) \\
\hline 34 & Phyllostomus hastatus (Pallas, 1767) & $\mathrm{LC}$ & DA & \\
\hline & Phyllostomus hastatus panamensis Allen, 1904 & & & Santos, Aguirre, Vásquez y Ortega (2003) \\
\hline 35 & Tonatia saurophila Koopman y Williams, 1951 & $\mathrm{LC}$ & DA & \\
\hline & Tonatia saurophila bakeri Williams, Willig y Reid, 1995 & & & Ramírez-Pulido et al. (2014) \\
\hline 36 & Trachops cirrhosus (Spix, 1823) & $\mathrm{LC}$ & DA & \\
\hline & Trachops cirrhosus coffini Felten, 1956 & & & Cramer, Willig y Jones (2001) \\
\hline 37 & Trinycteris nicefori (Sanborn, 1949) & $\mathrm{LC}$ & DA & \\
\hline
\end{tabular}


Tabla 1 (continuación)

\begin{tabular}{|c|c|c|c|c|}
\hline Núm. & Categoría taxonómica & UICN & NEn & Fuente \\
\hline 38 & $\begin{array}{l}\text { Vampyrum spectrum (Linnaeus, 1758) } \\
\text { Vampyrum spectrum nelsoni Goldman, } 1917 \\
\text { Subfamilia Carolliinae Miller, } 1924\end{array}$ & NT & DA & Navarro y Wilson (1982) \\
\hline 39 & Carollia castanea $\mathrm{H}$. Allen, 1890 & $\mathrm{LC}$ & DA & \\
\hline 40 & $\begin{array}{l}\text { Carollia perspicillata (Linnaeus, 1758) } \\
\text { Carollia perspicillata azteca Saussure, } 1860\end{array}$ & $\mathrm{LC}$ & DA & Cloutier y Thomas (1992) \\
\hline 41 & Carollia sowelli Baker, Solari y Hoffman, 2002 & $\mathrm{LC}$ & ER & \\
\hline 42 & $\begin{array}{l}\text { Carollia subrufa (Hahn, 1905) } \\
\text { Subfamilia Stenodermatinae Gervais, } 1856 \\
\text { Tribu Sturnirini Miller, } 1907\end{array}$ & $\mathrm{LC}$ & ER & \\
\hline 43 & Sturnira parvidens Goldman, 1917 & $\mathrm{NE}$ & ER & \\
\hline 44 & $\begin{array}{l}\text { Sturnira hondurensis Goodwin, } 1940 \\
\text { Sturnira hondurensis hondurensis Goodwin, } 1940 \\
\text { Tribu Stenodermatini Gervais, } 1856\end{array}$ & $\mathrm{NE}$ & ER & Ramírez-Pulido et al. (2014) \\
\hline 45 & $\begin{array}{l}\text { Artibeus aztecus K. Andersen, } 1906 \\
\text { Artibeus aztecus minor Davis, } 1969\end{array}$ & $\mathrm{LC}$ & DA & Webster y Knox-Jones (1982a) \\
\hline 46 & $\begin{array}{l}\text { Artibeus jamaicensis Leach, } 1821 \\
\text { Artibeus jamaicensis paulus Davis, } 1970 \\
\text { Artibeus jamaicensis richardsoni Allen, } 1908 \\
\text { Artibeus jamaicensis yucatanicus Andersen, } 1908\end{array}$ & $\mathrm{LC}$ & DA & $\begin{array}{l}\text { Ortega y Castro-Arellano (2001) } \\
\text { Ortega y Castro-Arellano (2001) } \\
\text { Ortega y Castro-Arellano (2001) }\end{array}$ \\
\hline 47 & $\begin{array}{l}\text { Artibeus lituratus (Olfers, 1818) } \\
\text { Artibeus lituratus palmarum Allen y Chapman, } 1897\end{array}$ & $\mathrm{LC}$ & DA & Marques-Aguiar (2007) \\
\hline 48 & $\begin{array}{l}\text { Artibeus phaeotis (Miller, 1902) } \\
\text { Artibeus phaeotis palatinus Davis, } 1970 \\
\text { Artibeus phaeotis phaeotis (Miller, 1902) }\end{array}$ & $\mathrm{LC}$ & DA & $\begin{array}{l}\text { Timm }(1985) \\
\text { Timm }(1985)\end{array}$ \\
\hline 49 & $\begin{array}{l}\text { Artibeus toltecus (Saussure, 1860) } \\
\text { Artibeus toltecus hesperus Davis, } 1969 \\
\text { Artibeus toltecus toltecus (Saussure, 1860) }\end{array}$ & $\mathrm{LC}$ & DA & $\begin{array}{l}\text { Webster y Knox-Jones (1982b) } \\
\text { Webster y Knox-Jones (1982b) }\end{array}$ \\
\hline 50 & Artibeus watsoni Thomas, 1901 & $\mathrm{LC}$ & ER & \\
\hline 51 & $\begin{array}{l}\text { Centurio senex Gray, } 1842 \\
\text { Centurio senex senex Gray, } 1842\end{array}$ & $\mathrm{LC}$ & DA & Snow, Knox-Jones y Webster (1980) \\
\hline 52 & $\begin{array}{l}\text { Chiroderma salvini Dobson, } 1878 \\
\text { Chiroderma salvini salvini Handley, } 1966 \\
\text { Chiroderma salvini scopaeum Handley, } 1966\end{array}$ & $\mathrm{LC}$ & DA & $\begin{array}{l}\text { Gardner (2007) } \\
\text { Gardner (2007) }\end{array}$ \\
\hline 53 & $\begin{array}{l}\text { Chiroderma villosum Peters, } 1860 \\
\text { Chiroderma villosum jesupi J.A. Allen, } 1900\end{array}$ & $\mathrm{LC}$ & DA & Gardner (2007) \\
\hline 54 & Enchisthenes hartii (Thomas, 1892) & $\mathrm{LC}$ & DA & \\
\hline 55 & Platyrrhinus helleri (Peters, 1866) & $\mathrm{LC}$ & DA & \\
\hline 56 & Uroderma convexum Lyon, 1902 & $\mathrm{NE}$ & ER & \\
\hline 57 & Uroderma davisi Baker y McDaniel, 1972 & $\mathrm{NE}$ & ER & \\
\hline 58 & Uroderma magnirostrum Davis, 1968 & $\mathrm{LC}$ & DA & \\
\hline 59 & Vampyressa thyone Thomas, 1909 & $\mathrm{LC}$ & DA & \\
\hline 60 & $\begin{array}{l}\text { Vampyrodes major G.M. Allen, } 1908 \\
\text { Familia Mormoopidae Saussure, } 1860\end{array}$ & $\mathrm{LC}$ & DA & \\
\hline 61 & $\begin{array}{l}\text { Mormoops megalophylla (Peters, 1864) } \\
\text { Mormoops megalophylla megalophylla Peters, } 1864\end{array}$ & $\mathrm{LC}$ & DA & Rezsutek y Cameron (1993) \\
\hline 62 & $\begin{array}{l}\text { Pteronotus davyi Gray, } 1838 \\
\text { Pteronotus davyi fulvus (Thomas, 1892) }\end{array}$ & $\mathrm{LC}$ & DA & Adams (1989) \\
\hline 63 & Pteronotus gymnonotus Natterer, 1843 & $\mathrm{LC}$ & DA & \\
\hline 64 & Pteronotus mesoamericanus Smith, 1972 & NE & ER & \\
\hline 65 & $\begin{array}{l}\text { Pteronotus personatus (Wagner, 1843) } \\
\text { Pteronotus personatus psilotis (Dobson, 1878) } \\
\text { Familia Noctilionidae Gray, } 1821\end{array}$ & $\mathrm{LC}$ & DA & De la Torre y Medellín (2010) \\
\hline 66 & $\begin{array}{l}\text { Noctilio albiventris Desmarest, } 1818 \\
\text { Noctilio albiventris minor Osgood, } 1919\end{array}$ & $\mathrm{LC}$ & DA & Hood y Pitocchelli (1983) \\
\hline 67 & $\begin{array}{l}\text { Noctilio leporinus (Linnaeus, 1758) } \\
\text { Noctilio leporinus mastivus (Vahl, 1797) } \\
\text { Familia Thyropteridae Miller, } 1907\end{array}$ & $\mathrm{LC}$ & DA & Hood y Knox-Jones (1984) \\
\hline 68 & $\begin{array}{l}\text { Thyroptera tricolor Spix, } 1823 \\
\text { Thyroptera tricolor albiventer (Tomes, 1856) } \\
\text { Familia Natalidae Gray, } 1866\end{array}$ & $\mathrm{LC}$ & DA & Wilson y Findley (1977) \\
\hline 69 & $\begin{array}{l}\text { Natalus mexicanus Miller, } 1902 \\
\text { Familia Molossidae Gervais, } 1856 \\
\text { Subfamilia Molossinae Gervais, } 1856\end{array}$ & $\mathrm{LC}$ & DA & \\
\hline
\end{tabular}


Tabla 1 (continuación)

\begin{tabular}{|c|c|c|c|c|}
\hline Núm. & Categoría taxonómica & UICN & NEn & Fuente \\
\hline \multirow[t]{2}{*}{70} & Eumops auripendulus (Shaw, 1800) & $\mathrm{LC}$ & DA & \\
\hline & Eumops auripendulus auripendulus (Shaw, 1800) & & & Best, Hunt, McWilliams y Smith (2002) \\
\hline 71 & Eumops ferox (Gundlach, 1961) & $\mathrm{NE}$ & DA & \\
\hline 72 & Molossus aztecus Saussure, 1860 & $\mathrm{LC}$ & DA & \\
\hline 73 & Molossus molossus (Pallas, 1766) & $\mathrm{LC}$ & DA & \\
\hline \multirow[t]{2}{*}{74} & Molossus rufus E. Geoffroy, 1805 & $\mathrm{LC}$ & DA & \\
\hline & Molossus rufus nigricans Miller, 1902 & & & Ramírez-Pulido et al. (2014) \\
\hline \multirow[t]{2}{*}{75} & Molossus sinaloe J.A. Allen, 1906 & $\mathrm{LC}$ & DA & \\
\hline & Molossus sinaloe sinaloe J.A. Allen, 1906 & & & Ramírez-Pulido et al. (2014) \\
\hline \multirow[t]{2}{*}{76} & Nyctinomops laticaudatus (E. Geoffroy, 1805) & $\mathrm{LC}$ & DA & \\
\hline & Nyctinomops laticaudatus yucatanicus Miller, 1902 & & & Ávila-Flores, Flores-Martínez y Ortega (2002) \\
\hline \multirow[t]{2}{*}{77} & Promops centralis Thomas, 1915 & $\mathrm{LC}$ & DA & \\
\hline & Promops centralis centralis Thomas, 1915 & & & Ramírez-Pulido et al. (2014) \\
\hline \multirow[t]{5}{*}{78} & Tadarida brasiliensis (I. Geoffroy, 1824) & $\mathrm{LC}$ & DA & \\
\hline & Tadarida brasiliensis intermedia Shamel, 1931 & & & Wilkins (1989) \\
\hline & Familia Vespertilionidae Gray, 1821 & & & \\
\hline & Subfamilia Vespertilioninae Gray, 1821 & & & \\
\hline & Tribu Eptesicini Volleth y Heller, 1994 & & & \\
\hline \multirow[t]{2}{*}{79} & Eptesicus brasiliensis (Desmarest, 1819) & $\mathrm{LC}$ & DA & \\
\hline & Eptesicus brasiliensis brasiliensis (Desmarest, 1819) & & & Ramírez-Pulido et al. (2014) \\
\hline \multirow[t]{2}{*}{80} & Eptesicus furinalis (D’Orbigny, 1847) & $\mathrm{LC}$ & DA & \\
\hline & Eptesicus furinalis gaumeri (J.A. Allen, 1897) & & & Mies, Kurta y King (1996) \\
\hline \multirow[t]{3}{*}{81} & Eptesicus fuscus (Beauvois, 1796) & $\mathrm{LC}$ & DA & \\
\hline & Eptesicus fuscus miradorensis (H. Allen, 1866) & & & Kurta y Baker (1990) \\
\hline & Tribu Lasiurini Tate, 1942 & & & \\
\hline \multirow[t]{2}{*}{82} & Lasiurus blossevillii (Lesson y Garnot, 1826) & $\mathrm{LC}$ & DA & \\
\hline & Lasiurus blossevillii frantzii (W. Peters, 1870) & & & Gardner y Handley (2007) \\
\hline \multirow[t]{2}{*}{83} & Lasiurus cinereus (Palisot de Beauvois, 1796) & $\mathrm{LC}$ & DA & \\
\hline & Lasiurus cinereus cinereus (Palisot de Beauvois, 1796) & & & Shump y Shump (1982) \\
\hline \multirow[t]{2}{*}{84} & Lasiurus ega (Gervais, 1856$)$ & $\mathrm{LC}$ & DA & \\
\hline & Lasiurus ega panamensis (Thomas, 1901) & & & Kurta y Lehr (1995) \\
\hline \multirow[t]{2}{*}{85} & Lasiurus intermedius $\mathrm{H}$. Allen, 1862 & $\mathrm{LC}$ & DA & \\
\hline & $\begin{array}{l}\text { Lasiurus intermedius intermedius H. Allen, } 1862 \\
\text { Tribu Nycticeiini Gervais, } 1855\end{array}$ & & & Webster, Knox-Jones y Baker (1980) \\
\hline 86 & Rhogeessa aeneus Goodwin, 1958 & $\mathrm{LC}$ & ER & \\
\hline \multirow{3}{*}{$\begin{array}{l}81 \\
88\end{array}$} & Rhogeessa bickhami & $\mathrm{NE}$ & ER & Baird et al., 2012 \\
\hline & Rhogeessa menchuae & $\mathrm{NE}$ & $\mathrm{ER}$ & Baird et al., 2012 \\
\hline & Tribu inc. sed. & & & Hoofer y van den Bussche (2003) \\
\hline \multirow[t]{3}{*}{89} & Perimyotis subflavus (F. Cuvier, 1832) & $\mathrm{LC}$ & DA & \\
\hline & Perimyotis subflavus veraecrucis (Ward, 1891) & & & Fujita y Kunz (1984) \\
\hline & Subfamilia Antrozoinae Miller, 1897 & & & \\
\hline \multirow[t]{2}{*}{90} & Bauerus dubiaquercus (Van Gelder, 1959) & NT & ER & \\
\hline & Subfamilia Myotinae Tate, 1942 & & & \\
\hline 91 & Myotis albescens (E. Geoffroy, 1806) & $\mathrm{LC}$ & DA & \\
\hline \multirow{3}{*}{$\begin{array}{l}92 \\
93\end{array}$} & Myotis auriculus Baker y Stains, 1955 & NA & NA & \\
\hline & Myotis californicus (Audubon y Bachman, 1842) & $\mathrm{LC}$ & DA & \\
\hline & Myotis californicus mexicanus Saussure, 1860 & & & Simpson (1993) \\
\hline 94 & Myotis cobanensis & DD & $\mathrm{EN}$ & Goodwin, 1955 \\
\hline 95 & Myotis elegans Hall, 1962 & $\mathrm{LC}$ & ER & \\
\hline 96 & Myotis fortidens Miller y Allen, 1928 & $\mathrm{LC}$ & DA & \\
\hline & Myotis fortidens fortidens Miller y Allen, 1928 & & & Ramírez-Pulido et al. (2014) \\
\hline 97 & Myotis keaysi J.A. Allen, 1914 & $\mathrm{LC}$ & DA & \\
\hline & Myotis keaysi pilosatibialis LaVal, 1973 & & & Hernández-Meza, Domínguez-Castellanos y Ortega (2005) \\
\hline 98 & Myotis nigricans (Schinz, 1821) & $\mathrm{LC}$ & DA & \\
\hline & Myotis nigricans nigricans (Schinz, 1821) & & & Wilson y LaVal (1974) \\
\hline 99 & Myotis thysanodes Miller, 1897 & $\mathrm{LC}$ & DA & \\
\hline & Myotis thysanodes aztecus Miller y G.M. Allen, 1928 & & & O'Farrell y Studier (1980) \\
\hline 100 & Myotis velifer (J.A. Allen, 1890) & $\mathrm{LC}$ & DA & \\
\hline & Myotis velifer velifer (J.A. Allen, 1890) & & & Fitch, Shump y Shump (1981) \\
\hline
\end{tabular}

UICN: estado de conservación según la Unión Internacional para la Conservación de la Naturaleza (acrónimos en inglés): DA: distribución amplia; DD: datos deficientes; NT: casi amenazado; ER: endémico regional; LC: preocupación menor; EN: endémico nacional; NA: no asignable; NE: no evaluado; VU: vulnerable. 
L. curasoae queda restringida a Sudamérica, isla Margarita y las Antillas Holandesas (Simmons, 2005). Por último, se incluyen a Micronycteris minuta (Phyllostominae) y Carollia castanea (Carolliinae), cuyos registros en el país están basados en recolectas recientes, con ejemplares depositados en la colección del Museo de Historia Natural, Universidad de San Carlos de Guatemala.

\section{Familia Mormoopidae Saussure, 1860}

Para esta familia, en el caso de Pteronotus parnellii (Gray, 1843) que previamente era considerada de distribución amplia (Miller, Reid, Arroyo-Cabrales, Cuarón y de Grammont, 2008), Clare et al. (2013) presentan evidencia de que se trata de un complejo de especies, lo cual infieren mediante análisis moleculares y morfológicos, aunque también variaciones en las frecuencias de ecolocalización. Estos autores consideran a las poblaciones de $P$. parnellii en Centroamérica diferenciadas de las de Sudamérica y sugieren asignarles como nombre apropiado P. mesoamericanus Smith, 1972.

\section{Familia Natalidae Gray, 1866}

Para esta familia, se da el reconocimiento de Natalus mexicanus Miller, 1902 que en Guatemala previamente era identificada como N. stramineus Gray, 1838 (MacCarthy y Pérez, 2006). Históricamente, el nombre $N$. stramineus era asignado a las poblaciones de este género en el Neotrópico (Tejedor, 2006); sin embargo, después de la controversia sobre el origen del holotipo y con base en evidencia morfológica, $N$. stramineus queda restringido a las Antillas Menores y las poblaciones continentales de esta especie previa representan especies alopátricas con $N$. mexicanus reconocido desde el norte de México hasta Panamá (Tejedor, 2006, 2011).

\section{Familia Molossidae Gervais, 1856}

Para esta familia, Dolan (1989) llevó a cabo un análisis del género Molossus donde comprueba que la especie previamente reconocida como $M$. ater E. Geoffroy, 1805 debía ser reasignada a $M$. rufus E. Geoffroy, 1805. Además, MacCarthy y Pérez (2006) reconocían a la subespecie M. molossus aztecus Saussure, 1860 y en esta lista, de acuerdo con Dolan (1989) y Simmons (2005), se incluye en la categoría de especie como M. aztecus Saussure, 1860; en Guatemala se tiene un registro en Aguacatán, Huehuetenango (Dolan, 1989). Asimismo, McDonough et al. (2008) reconocen a Eumops glaucinus como un complejo en el cual E. ferox (Gundlach, 1961) es la única especie reconocida para México, Centroamérica y el Caribe, mientras que $E$. glaucinus, enlistada por Pérez et al. (2012), queda restringida a Sudamérica.

\section{Familia Vespertilionidae Gray, 1821}

Para esta familia, Myotis cobanensis (Myotinae) es la única especie de murciélago endémico nacional (MacCarthy y Pérez, 2006). Su recolecta data del año 1946 y fue descrita en 1955 por Goodwin como una subespecie, M. velifer cobanensis, a partir de un solo ejemplar de una única localidad registrada en Cobán, Alta Verapaz (De la Torre, 1958; Goodwin, 1955). Posteriormente, De la Torre (1958) propone elevarla a la categoría taxonómica de especie. Debido a la antigüedad de su registro, a su incertidumbre taxonómica y a datos deficientes para definir su estado de conservación (Cajas y Miller, 2008), se consideran necesarios esfuerzos para esclarecer su situación taxonómica. Asimismo, para Myotis auriculus (Myotinae) se reconocen 2 subespecies: M. auriculus auriculus Baker y Stains, 1955 y $M$. auriculus apache Hoffmeister y Krutzsch, 1955 (Warner, 1982). En Guatemala, esta especie se conoce de un único registro en San Jorge Muxbal, San José Pinula (Hoffman, Knox-Jones y Campbell, 1987); sin embargo, Hoffman et al. (1987) indican que dicho ejemplar se diferencia de la subespecie más cercana geográficamente (M. a. auriculus) y proponen que representa una población sureña disjunta y aislada del istmo de Tehuantepec; mientras que Arroyo-Cabrales, Miller, Reid, Cuarón y de Grammont (2008) las consideran una misma. Hoffman et al. (1987) indican la importancia de más ejemplares para determinar la situación taxonómica y el rango geográfico de esta población, por lo que en esta lista se incluye en la categoría NA en lo que respecta a estado de conservación y nivel de endemismo.

Por otro lado, Rhogeessa tumida H. Allen, 1866 (Vespertilioninae, Nycticeiini), anteriormente incluida en la lista de MacCarthy y Pérez (2006), se trata de un complejo de especies reconocidas como linajes, de las cuales $R$. bickhami Baird et al., 2012 y R. menchuae Baird et al., 2012 están registradas en el país (Baird et al., 2012). Para la primera, se tiene una recolecta en el oeste del Valle del Motagua y, para la segunda, en río Vega Grande, Los Amates, Izabal (Baird et al., 2012). Por su parte, para Pipistrellus subflavus (F. Cuvier, 1832) (Vespertilioninae, Pipistrellini), única especie Neotropical de este género, se validó el nombre Perimyotis subflavus (F. Cuvier, 1832) (Vespertilioninae, inc. sed., Hoofer y van den Bussche, 2003) al comprobarse su polifilia (Hoofer, van den Bussche y Horacek, 2006; Solari y Martínez-Arias, 2014). Por último, la subespecie Eptesicus brasiliensis andinus J.A. Allen, 1914 incluida en la lista de MacCarthy y Pérez (2006), queda restringida a Sudamérica, tratándose las poblaciones en México y Centroamérica de E. b. brasiliensis (Desmarest, 1819) (Ramírez-Pulido et al., 2014; Simmons, 2005).

Desde 2005, la clasificación taxonómica de los murciélagos neotropicales ha experimentado cambios considerables, con un incremento neto de 8 géneros y 75 especies (Solari y MartínezArias, 2014). A medida que aumente el esfuerzo de investigación y en los estudios taxonómicos se integren datos genéticos, morfológicos y biogeográficos, mejorará el conocimiento de la riqueza de especies en la región, por lo que las listas deben ser actualizadas periódicamente para ofrecer información precisa, con implicaciones en los programas de conservación (Solari y Martínez-Arias, 2014).

\section{Agradecimientos}

Agradecemos a B. Rodríguez, coordinador de la «Estrategia Centroamericana para la Conservación de los Murciélagos», la 
oportunidad de participar en talleres de capacitación en sistemática, ecología y conservación de murciélagos. A E. Leiva del Programa para la Conservación de los Murciélagos de Guatemala (PCMG), su apoyo en la revisión del estado taxonómico y categorías de amenaza de las especies de murciélagos de Guatemala. Por último, queremos expresar nuestro agradecimiento a 2 revisores anónimos, cuyos comentarios ayudaron a mejorar este manuscrito.

\section{Referencias}

Adams, J. K. (1989). Pteronotus davyi. Mammalian Species, 346, 1-5.

Alonso-Mejía, A. y Medellín, R. A. (1991). Micronycteris megalotis. Mammalian Species, 376, 1-6.

Álvarez, J., Willig, M. R., Knox-Jones, J., Jr. y Webster, W. D. (1991). Glossophaga soricina. Mammalian Species, 379, 1-7.

Arroyo-Cabrales, J. y Knox-Jones, J., Jr. (1988). Balantiopteryx plicata. Mammalian Species, 301, 1-4.

Arroyo-Cabrales, J., Miller, B., Reid, F., Cuarón, A. D. y de Grammont, P. C. (2008). Myotis auriculus. En The IUCN red list of threatened species 2008: e.T14145A4409132. Recuperado 2 Oct 2015 de: www.iucnredlist.org

Ávila-Flores, R., Flores-Martínez, J. J. y Ortega, J. (2002). Nyctinomops laticaudatus. Mammalian Species, 697, 1-6.

Baird, A. B., Marchán-Rivadeneira, M. R., Pérez, S. G. y Baker, R. J. (2012). Morphological analysis and description of two new species of Rhogeessa (Chiroptera: Vespertilionidae) from the Neotropics. Occasional Papers, Museum of Texas Tech University, 307, 1-25.

Best, T. L., Hunt, J. L., McWilliams, L. A. y Smith, K. G. (2002). Eumops auripendulus. Mammalian Species, 708, 1-5.

Cajas, J. y Miller, B. (2008). Myotis cobanensis. En The IUCN red list of threatened species 2008: e.T14154A4411191. Recuperado 4 Jul 2015 de: www.iucnredlist.org

Castro-Arellano, I. y Marce, E. (2014). Greater dog-like bat. En G. Ceballos (Ed.), Mammals of Mexico (pp. 673-674). Baltimore, Maryland: Johns Hopkins University Press.

Ceballos, G. y Medellín, R. A. (1988). Diclidurus albus. Mammalian Species, $316,1-4$.

Clare, E. L., Adams, A. M., Maya-Simões, A. Z., Eger, J. L., Hebert, P. D. N. y Fenton, M. B. (2013). Diversification and reproductive isolation: cryptic species in the only New World high-duty cycle bat, Pteronotus parnellii. BMC Evolutionary Biology, 13, 26.

Cloutier, D. y Thomas, D. W. (1992). Carollia perspicillata. Mammalian Species, 417, 1-9.

Cramer, M. J., Willig, M. R. y Jones, C. (2001). Trachops cirrhosus. Mammalian Species, 656, 1-6.

De la Torre, L. (1958). The status of the bat Myotis velifer cobanensis Goodwin. Proceedings of the Biological Society of Washington, 71, 167-170.

De la Torre, A. y Medellín, R. A. (2010). Pteronotus davyi. Mammalian Species, $42,244-250$.

Ditchfield, A. D. (2000). The comparative phylogeography of Neotropical mammals: patterns of intraspecific mitochondrial DNA variation among bats contrasted to nonvolant small mammals. Molecular Ecology, 9, 1307-1318.

Dolan, P. G. (1989). Systematics of Middle American mastiff bats of the genus Molossus. Special Publications, The Museum of Texas Tech University, 29, $1-65$.

Fitch, J. H., Shump, K. A., Jr. y Shump, A. U. (1981). Myotis velifer. Mammalian Species, 149, 1-5.

Fujita, M. S. y Kunz, T. H. (1984). Pipistrellus subflavus. Mammalian Species, $228,1-6$.

Gardner, A. L. (2007). Genus Chiroderma W. Peters, 1860. En A. L. Gardner (Ed.), Mammals of South America. Marsupials, xenarthrans, shrews, and bats (pp. 321-326). Chicago: The University of Chicago Press.

Gardner, A. L. y Handley, O., Jr. (2007). Genus Lasiurus Gray, 1831. En A. L. Gardner (Ed.), Mammals of South America. Marsupials, xenarthrans, shrews, and bats (pp. 457-468). Chicago: The University of Chicago Press.
Goodwin, G. G. (1955). Mammals from Guatemala, with the description of a new little brown bat. American Museum Novitates, 1744, 1-5.

Greenhall, A. M., Joermann, G., Schmidt, U. y Seidel, M. R. (1983). Desmodus rotundus. Mammalian Species, 202, 1-6.

Greenhall, A. M., Schmidt, U. y Joermann, G. (1984). Diphylla ecaudata. Mammalian Species, 227, 1-3.

Hernández-Meza, B., Domínguez-Castellanos, Y. y Ortega, J. (2005). Myotis keaysi. Mammalian Species, 785, 1-3.

Hoffman, R. S., Knox-Jones, J., Jr. y Campbell, J. A. (1987). First record of Myotis auriculus from Guatemala. The Southwestern Naturalist, 32, 391.

Hood, C. S. y Knox-Jones, J., Jr. (1984). Noctilio leporinus. Mammalian Species, $216,1-7$.

Hood, C. S. y Pitocchelli, J. (1983). Noctilio albiventris. Mammalian Species, 197, 1-5.

Hoofer, S. R., Solari, S., Larsen, P. A., Bradley, R. D. y Baker, R. J. (2008). Phylogenetics of the fruit-eating bats (Phyllostomidae: Artibeina) inferred from mitochondrial DNA sequences. Occasional Papers, Museum of Texas Tech University, 277, 1-15.

Hoofer, S. R. y van den Bussche, R. A. (2003). Molecular phylogenetics of the chiropteran family Vespertilionidae. Acta Chiropterologica, 5, 1-63.

Hoofer, S. R., van den Bussche, R. A. y Horacek, I. (2006). Generic status of the American pipistrelles (Vespertilionidae) with description of a new genus. Journal of Mammalogy, 87, 981-992.

Knox-Jones, J., Jr. y Homan, J. A. (1974). Hylonycteris underwoodi. Mammalian Species, 32, 1-2.

Kurta, A. y Baker, R. H. (1990). Eptesicus fuscus. Mammalian Species, 356, $1-10$.

Kurta, A. y Lehr, G. C. (1995). Lasiurus ega. Mammalian Species, 515, 1-7.

Kwiecinski, G. G. (2006). Phyllostomus discolor. Mammalian Species, 801, $1-11$.

MacCarthy, T. J. y Pérez, S. G. (2006). Land and freshwater mammals of Guatemala: faunal documentation and diversity. En E. Cano (Ed.), Biodiversidad de Guatemala (pp. 625-674). Guatemala: Universidad del Valle de Guatemala.

Mantilla-Meluk, H. (2014). Defining species and species boundaries in Uroderma (Chiroptera: Phyllostomidae) with a description of a new species. Occasional Papers, Museum of Texas Tech University, 325, 1-25.

Marques-Aguiar, S. A. (2007). Genus Artibeus Leach, 1821. En A. L. Gardner (Ed.), Mammals of South America. Marsupials, xenarthrans, shrews, and bats (pp. 301-321). Chicago: The University of Chicago Press.

McDonough, M. M., Ammerman, L. K., Timm, R. M., Genoways, H. H., Larsen, P. A. y Baker, R. J. (2008). Speciation within bonneted bats (genus Eumops): The complexity of morphological, mitochondrial, and nuclear data sets in systematics. Journal of Mammalogy, 89, 1306-1315.

Medellín, R. A. (1989). Chrotopterus auritus. Mammalian Species, 343, 1-5.

Méndez, C. (2008). Diversidad faunística de Guatemala. En C. Azurdia, F. García, y M. M. Ríos (Eds.), Guatemala y su biodiversidad: un enfoque histórico, cultural, biológico y económico (pp. 231-259). Guatemala: Oficina Técnica de Biodiversidad, Consejo Nacional de Áreas Protegidas.

Mies, R., Kurta, A. y King, D. G. (1996). Eptesicus furinalis. Mammalian Species, 526, 1-7.

Miller, B., Reid, F., Arroyo-Cabrales, J., Cuarón, A.D. y de Grammont, P.C. (2008). Pteronotus parnellii. En The IUCN red list of threatened species 2008: e.T18708A8506002. Recuperado 29 May 2015 de: www.iucnredlist.org

Navarro, D. y Wilson, D. E. (1982). Vampyrum spectrum. Mammalian Species, 184, 1-4.

O'Farrell, M. J. y Studier, E. H. (1980). Myotis thysanodes. Mammalian Species, $137,1-5$.

Ortega, J. y Alarcón, D. I. (2008). Anoura geoffroyi. Mammalian Species, 818, $1-7$.

Ortega, J. y Castro-Arellano, I. (2001). Artibeus jamaicensis. Mammalian Species, 662, 1-9.

Pérez, S. G., López, J. E. y MacCarthy, T. J. (2012). Five new records of bats for Guatemala, with comments on the checklist of the country. Chiroptera Neotropical, 18, 1106-1110. 
Ramírez-Pulido, J., González-Ruiz, N., Gardner, A. L. y Arroyo-Cabrales, J. (2014). List of recent land mammals of Mexico, 2014. Special Publications, Museum of Texas Tech University, 63, 1-69.

Redondo, R. A. F., Brina, L. P. S., Silva, R. F., Ditchfield, A. D. y Santos, F. R. (2008). Molecular systematics of the genus Artibeus (Chiroptera: Phyllostomidae). Molecular Phylogenetics and Evolution, 49, 44-58.

Rezsutek, M. y Cameron, G. N. (1993). Mormoops megalophylla. Mammalian Species, 448, 1-5.

Santos, M., Aguirre, L. F., Vásquez, L. B. y Ortega, J. (2003). Phyllostomus hastatus. Mammalian Species, 722, 1-6.

Shump, K. A., Jr. y Shump, A. U. (1982). Lasiurus cinereus. Mammalian Species, 185, 1-5.

Simmons, N. B. (2005). Order Chiroptera. En D. E. Wilson y D. M. Reeder (Eds.), Mammal species of the world: a taxonomic and geographic reference (Tercera edición, pp. 312-529). Baltimore: Johns Hopkins Press.

Simpson, M. R. (1993). Myotis californicus. Mammalian Species, 428, 1-4.

Snow, J. L., Knox-Jones, J., Jr. y Webster, W. D. (1980). Centurio senex. Mammalian Species, 138, 1-3.

Solari, S. y Martínez-Arias, V. (2014). Cambios recientes en la sistemática y taxonomía de murciélagos neotropicales (Mammalia: Chiroptera). Therya, $5,167-196$

Tejedor, A. (2006). The type locality of Natalus stramineus (Chiroptera: Natalidae): Implications for the taxonomy and biogeography of the genus Natalus. Acta Chiropterologica, 8, 361-380.

Tejedor, A. (2011). Systematics of the funnel-eared bats (Chiroptera: Natalidae). Bulletin of the American Museum of Natural History, 353, 1-140.

Timm, R. M. (1985). Artibeus phaeotis. Mammalian Species, 235, 1-6.

UICN (Unión Internacional para la Conservación de la Naturaleza). (2015). The IUCN red list of threatened species. Versión 2015.3. Recuperado de: http://www.iucnredlist.org
Velazco, P. M., Aires, C. C., Carmignotto, A. P. y Bezerra, A. M. R. (2010), Mammalia, Chiroptera, Phyllostomidae, Vampyrodes caraccioli (Thomas, 1889): range extension and revised distribution map. Check List, 6, 49-51.

Velazco, P. M. y Patterson, B. D. (2013). Diversification of the yellow-shouldered bats, genus Sturnira (Chiroptera, Phyllostomidae), in the New World tropics. Molecular Phylogenetics and Evolution, 68, 683-698.

Velazco, P. M. y Patterson, B. D. (2014). Two new species of yellow-shouldered bats, genus Sturnira Gray, 1842 (Chiroptera, Phyllostomidae) from Costa Rica, Panama and western Ecuador. ZooKeys, 402, 43-66.

Velazco, P. M. y Simmons, N. B. (2011). Systematics and taxonomy of great striped-faced bats of the genus Vampyrodes Thomas, 1900 (Chiroptera: Phyllostomidae). American Museum Novitates, 3710, 1-35.

Warner, R. M. (1982). Myotis auriculus. Mammalian Species, 191, 1-3.

Webster, W. D. y Knox-Jones, J., Jr. (1982a). Artibeus aztecus. Mammalian Species, 177, 1-3.

Webster, W. D. y Knox-Jones, J., Jr. (1982b). Artibeus toltecus. Mammalian Species, 178, 1-3.

Webster, W. D. y Knox-Jones, J., Jr. (1993). Glossophaga commissarisi. Mammalian Species, 446, 1-4.

Webster Wm., D., Knox-Jones, J., Jr. y Baker, R. J. (1980). Lasiurus intermedius. Mammalian Species, 132, 1-3.

Wilkins, K. T. (1989). Tadarida brasiliensis. Mammalian Species, 331, 1-10.

Williams, S. L. y Genoways, H. H. (2007). Subfamily Phyllostominae. En A. L. Gardner (Ed.), Mammals of South America. Marsupials, xenarthrans, shrews, and bats (pp. 255-299). Chicago: The University of Chicago Press.

Willis, K. B., Willig, M. R. y Knox-Jones, J., Jr. (1990). Vampyrodes caraccioli. Mammalian Species, 359, 1-4.

Wilson, D. E. y Findley, J. S. (1977). Thyroptera tricolor. Mammalian Species, 71, 1-3.

Wilson, D. E. y LaVal, R. K. (1974). Myotis nigricans. Mammalian Species, 39 $1-3$. 